\title{
An interview with Professor Raj Reddy on Web Intelligence (WI) and Computational Social Science (CSS)
}

\author{
Ning Zhong a,b,*, Jiming Liu ${ }^{\mathrm{c}}$, Yong Shi ${ }^{\mathrm{d}, \mathrm{e}, \mathrm{f}}$ and Yiyu Yao ${ }^{\mathrm{g}}$ \\ ${ }^{a}$ Department of Life Science and Informatics, Maebashi Institute of Technology, Maebashi 371-0816, Japan \\ ${ }^{\mathrm{b}}$ International WIC Institute, Beijing University of Technology, Beijing 100022, China \\ ${ }^{\mathrm{c}}$ Department of Computer Science, Hong Kong Baptist University, Hong Kong \\ ${ }^{\mathrm{d}}$ School of Economics and Management, University of Chinese Academy of Sciences, Beijing 100190, China \\ e The Key Laboratory of Big Data Mining and Knowledge Management, Chinese Academy of Sciences, Beijing \\ 100190, China \\ ${ }^{\mathrm{f}}$ Research Center on Fictitious Economy and Data Science, Chinese Academy of Sciences, Beijing 100190, China \\ $\mathrm{g}$ Department of Computer Science, University of Regina, Regina, Saskatchewan, Canada
}

\section{Background}

Professor Dabbala Rajagopal "Raj" Reddy (born 13 June 1937) is an Indian-American computer scientist and has served on the faculty of Stanford and Carnegie Mellon for over 40 years. In 1994, he received the ACM Turing Award, the highest award in Computer Science, "for pioneering the design and construction of large scale artificial intelligence systems, demonstrating the practical importance and potential commercial impact of artificial intelligence technology." On 24 August 2017, Professor Raj Reddy gave a beautiful and eye-opening keynote talk entitled "The Ultimate Web Intelligence: Creating a Truly Humane Society Through Computational Social Science" at the IEEE/WIC/ACM International Conference on Web Intelligence in Leipzig, Germany. In this keynote, he stated that "Computer Science and Artificial Intelligence must embrace CSS [Computational Social Science] as the next frontier in Web Intelligence and WIC [Web Intelligence Consortium] needs to be at the forefront of inventing that future." His vision on Artificial Intelligence and Web Intelligence for creating a truly humane society is both thought-provoking and instrumental to bringing about new revolutions in the two related fields. We were very fortunate to have an in-

*Corresponding author. E-mail: zhong@maebashi-it.ac.jp. terview with Professor Reddy on 17 March 2018. We present excerpts from the interview by thematically organizing Professor Reddy's answers.

\section{Question 1: What is your vision of the future of AI?}

There are lots of speculations about the future going around. There are people like Elon Musk, and they say that robots are going to dominate the world and make us slaves. That's not going to happen! It won't happen in the next hundred years, and also won't happen in the next thousand years. What may happen? That is what I call the wise project in the AI, saying "We may have a robot-intensive world where we coexist with them." The robots are doing a lot of work. It's like having servants, right? The humanity has always had servants to do various things. In the future, there might be robots. And, the good thing is you don't have to worry about asking them to work 24 hours a day or all kinds of other things. You don't have to feed them, bath them and all that. But they need energy and electricity and so on. There will be a lot of positives, and some may be negatives about having robots. But that's the future I think, namely, robots and humans will coexist. They will do a lot of things that we normally don't want to do because it is boring or hazardous. My vision of 
the future $\mathrm{AI}$ is people and robots will coexist and be working together.

\section{Question 2: Can you comment on the cyber-physical-social (Web) Intelligence (i.e., AI for the connected world)?}

The main problem that's holding us back is to collect the data from the connected world, because using it is a privacy issue. The bottom line is, if we want to collect data from people, and use it in the Web Intelligence projects, then we must solve the privacy problem. And, the only way I can think of right now to solve the privacy problem is what is called opt in, that is, each individual voluntarily says "you can collect my data from my smartphone or whatever and use it." The Web Intelligence will potentially leverage a huge amount of social media data. It is possible to collect data from every possible service people land.

\section{Question 3: How could we further advance the field of computational social sciences via the route of Web Intelligence?}

Computational Social Science is a way of saying our fields, AI and Web Intelligence and so on, can do a lot of work to the society and solve the society problems. Now, everybody needs water, energy, food, health care, and shelter. Everybody needs the Internet. And, everybody needs all kinds of pharmaceutical medications and all kinds of things. I can give fifteen things everybody needs, whether they are rich or poor. And, if you consider putting each one of these things up, it will be helpful if you can collect data globally. Let us take water for an example. Web Intelligence has been an effective way for identifying potential problems such as water scarcity, identifying the cause of it, why it is happening, identifying possible solutions, and then actually doing it; namely, if you need a water tank, placing an order for a water tank on your behalf, even before you know there's a shortage of water. These kinds of things can be done in the future. That's what I mean by a computational social center point of societal issues.

You can take political science for the governance as an example. Different countries have different governing structures, and some of them believe in democracy. Every system has its own problems. In India, every time they want to build a road from a to $b$, it takes them ten years of going through public interest analysis and all kinds of things. In the end, only person says "Sorry, you can't use it and I need a hundred times and more money before I'll give you the thing." There is no perfect system. However, the issues of political systems can benefit from Web Intelligence.

Question 4: In your opinion, what could be the future impact of Web Intelligence? For instance, what would you see as the promises, opportunities and challenges of Web Intelligence in tackling real-world problems of social importance?

A lot of things about AI discovered over the last few years are that computers can be used for the discovery of scientific laws and so on. Herbert Simon demonstrated many of these discovered scientific laws, such as the role of emotion in decision-making. In general, what we are facing now is the big data revolution. There are lots of principles, laws, ideas, and concepts that can be discovered from the large amounts of data. A human being does not have the capacity to look at all the data and go through all the detail.

The amount of data from which you can actually infer things would be difficult for human beings to handle. And once you can infer those things, then you can use them to solve societal problems, and that's where the Web Intelligence comes in. If everybody opts in, it gives you the data. You have huge amounts of data to push into a database. And you can discover what is going on in the world and in every community, and it becomes possible to find solutions for the problems. This has not been possible before.

Actually, Web Intelligence becomes a central part of social science, anything that deals with society at large, people at large and communities at large. You've probably heard about smart cities, and they are all based on Web Intelligence. What is happening is that we are collecting data on a continuous basis within the city at a town or whatever. The data allows smart systems to notify the authorities by saying "There's a problem here." In the future, the system not only discovers our problems but also solves them. It tends to think and act on all feedback, not just sensing, not just monitoring, or not just diagnosing, but also preparing to solve problems. 
Question 5: Your concept of Personal Guardian Angels offers an important conceptual framework for future AI based systems. We would like to incorporate your framework to future Web Intelligence. What do think about this?

Basically, human beings will use agents in the future. Such agents are called cognition amplifiers or intelligence amplifiers. For example, an agent knows what you want to do, how to answer your emails, or how you want to pay your bills. It'll do these day in and day out routine tasks. The intelligent agent will just say "You need to pay the bill" and then it will send you a notification. Okay, those are called cognition amplifiers.

Then you have a set of agents that do things that you cannot do. Human beings have limited capacities for absorbing huge amounts of data and making inferences from there. It works like what Herbert Simon called satisficing. That is, human beings can face with huge amounts of data but simply will not use all the data and behave rationally. They will find a path that satisfies the problem at hand. And, that's why he got the Nobel Prize. They don't look for optimal solutions. They look for good enough solutions, because they just don't have the capacity to find the optimal solutions. In the future, there are all kinds of things, information and data globally available, that I as an individual can never hope to understand, master, and use effectively. That is where Web Intelligence and computational social science will play an important role.

The best example of this kind is supposing that it is going to be an earthquake, a tsunami or a typhoon. The government may broadcast on the TV, "There's going to be a tsunami in this area." But, what happens if I am not listening to that broadcast? If the Guardian Angel app is on your phone, which is always on, always working, and always learning not only from the data of the world, but also from other agents (other Guardian Angels), it will report by saying "I found the following information that the earthquake happened in Bolivia or Chili or somewhere." Another Guardian Angel may report, "I'm finding the same thing here and there that the earthquake is about to happen in Las Vegas." And so, they can do predictive inferences based on other agents' data. It's like a Facebook of Guardian Angels, just like there's a Facebook of people. Think a Facebook of Guardian Angels which are talking to each other and sharing information with each other.

The problem there is I might say "Hey, I don't want you to share my data with anybody else." And that's where the opportunity comes in, saying "If you want to benefit from the knowledge of everybody else, you need to link to share your data." And that's the way the traffic analysis company did in Israel which was then bought by Google for many billions of dollars. It is on a basis that everybody says "I benefit from it, therefore I'm willing to share the data," and so that everybody shares the data (big data), put up Guardian Angels. And then, this Guardian Angel for each kind of disaster (earthquakes or typhoon) is looking at all the global predictions. Based on what the world weather predictions were happening in there, and then using that to infer there's going to get a typhoon in your house in the next three hours or one hour? So, you'd better get into the basement.

Now that's where some interesting science has happened in Web Intelligence. They're all kinds of Web information published (e.g., on typhoon that's published for human consumption), and only a human being is able to read it and use it. But, in the future, this will be read by the Guardian Angels, not humans. They would infer from what has been posted and published. So, the page published must be visitable by humans and machines. Now, the technology doesn't exist, and there's a whole group of people that understand this need. They created a consortium based on schema, semantic labeling of Web Intelligence. This is a very important technical area for AI and Web Intelligence and so on; namely, machine readable Web pages. At the moment, most people that do HTML-based Web designers don't understand its importance. We must educate everyone for creating a machine understandable Web page and introduce a kind of certification process for Web developers. We need to have professional certification for Web designers, which includes the clear understanding of what it means to create machine readable Web pages.

In the future, you want robots to be able to read your things because human beings don't have the time. If you're interested in marketing, let your agent read all the agents. Your agent saves all the things you practice everyday. It is constantly looking at the best possible prices and locations and so on. And then it comes back and says "Hey, you normally buy coffee powder every two weeks, you can order it for $80 \%$ of the cost now as it is on sale." Yeah, then, I would say "Okay, go ahead." As another example, I want my machine, my Guardian Angels, to read all my emails, and reject most of the spams, and answer things about appointments, based on my past behavior, just like my secretary will do. 
Only one percent of the messages I have to see. All of that is not yet available in a Web intelligent system.

The personal Guardian Angels is a new kind of intelligent agents, not only the soft agents but also intelligent robots could be interconnected to the Internet. The Web pages are not only that the humans can read, but by using the semantic technology, they are also machine readable, and they can use the robots as the interface. In the Cyber-Physical systems, there are cyber robots and physical robots. And eighty percent of, or ninety percent of them, will not have a physical environment, and they are not physical robots, which do physical things like make my coffee or do other things. I think we need two kinds of different terminologies, to distinguish physical robots from virtual robots. For the virtual robots, they don't cost anything, because you just make more copies of them, while a physical robot every time you make it up, it cost you money. All I'm saying is both Guardian Angels and Cognitive Amplifiers are virtual agents. They always work and they don't need a physical presence, but they are on the cloud that knows everything. In particular, they can go look at my bank accounts every day. In the future, all backgrounds will be looked at by my agent, not me. They will warn me that there's no money.

I'm looking at the Web Intelligence and think we can also have a Web of Guardian Angels working together. That's what I call the Facebook of Guardian Angels, just like we have a Facebook of human beings, where we share information back and forth. If I have a Facebook of Guardian Angels, there will be the Guardian Angels that are dealing with earthquake. And so they all can share earthquake information back and forth and that can only happen if such a social system exists. The Facebook of Guardian Angels has lots of capabilities on communicating, sharing, publishing and liking, all those things. If I'm a Guardian Angel for earthquake, I can go and subscribe to all the earthquake agents on Facebook. Anybody may say, "If it's in my neighborhood, let me know."

\section{Question 6: What new roles would you suggest the Web Intelligence Consortium (WIC) plays?}

I think you'll come up with all those things mentioned earlier. Well, as you begin to implement the full potential of Web Intelligence, there'll be lots of specific things. I mentioned that you need machine readable Web pages. You need them to be professionally certified. There'll be probably ten or twenty or even a hundred such things that can be done in conjunction with Web Intelligence. The WIC could promote a culture of computational social science on the Web platform and play a leading role in creating the Facebook of Guardian Angels. For example, the WIC could provide standards for them, and then those standards will become global standards; everybody is expected to follow them. We need to develop computational social science and Web Intelligence technology to face the challenges of creating a truly humane society. 